\title{
A Temperature-Dependent Mechanistic Transition for Photoinduced Electron Transfer Modulated by Excited-State Vibrational Relaxation Dynamics
}

Youn K. Kang, Timothy V. Duncan, and Michael J. Therien*

Department of Chemistry, University of Pennsylvania,

Philadelphia, Pennsylvania 19104-6323 
Table S1. Temperature Dependent Experimental and Theoretical ET Rate Constants for the 2aZn Photoinduced Charge Separation Reaction in 2-MTHF Solvent.

\begin{tabular}{|c|c|c|c|c|c|c|c|c|c|c|}
\hline $\begin{array}{l}\mathrm{T} \\
\mathrm{K}\end{array}$ & $\begin{array}{c}1000 / \mathrm{T} \\
\mathrm{K}^{-1}\end{array}$ & $\begin{array}{l}k_{1}^{a} \\
\mathrm{ps}^{-1}\end{array}$ & $\begin{array}{l}k_{2}^{b} \\
\mathrm{ps}^{-1}\end{array}$ & $\begin{array}{l}k_{\text {class }}^{c} \\
\mathrm{ps}^{-1}\end{array}$ & $\begin{array}{c}k_{\text {semi50 }}{ }^{d} \\
\mathrm{ps}^{-1}\end{array}$ & $\begin{array}{c}k_{\text {semi90 }}^{e} \\
\mathrm{ps}^{-1}\end{array}$ & $\begin{array}{c}k_{\mathrm{SMB} 44 a^{f}} \\
\mathrm{ps}^{-1}\end{array}$ & $\begin{array}{c}k_{\mathrm{SMB} 44 b}{ }^{f} \\
\mathrm{ps}^{-1}\end{array}$ & $\begin{array}{c}k_{\mathrm{SMB} 95 a^{f}} \\
\mathrm{ps}^{-1}\end{array}$ & $\begin{array}{c}k_{\mathrm{SMB} 95 b}{ }^{f} \\
\mathrm{ps}^{-1}\end{array}$ \\
\hline 320 & 3.1 & 0.44 & & 0.40 & 0.40 & 1.31 & 0.41 & 0.41 & 1.96 & 1.86 \\
\hline 310 & 3.2 & 0.46 & & 0.42 & 0.41 & 1.32 & 0.42 & 0.42 & 1.96 & 1.86 \\
\hline 290 & 3.4 & 0.43 & & 0.44 & 0.42 & 1.35 & 0.41 & 0.42 & 1.95 & 1.86 \\
\hline 280 & 3.6 & 0.42 & & 0.46 & 0.42 & 1.36 & 0.41 & 0.42 & 1.94 & 1.87 \\
\hline 270 & 3.7 & 0.43 & & 0.47 & 0.42 & 1.37 & 0.41 & 0.41 & 1.93 & 1.87 \\
\hline 260 & 3.8 & 0.34 & & 0.49 & 0.42 & 1.38 & 0.41 & 0.41 & 1.90 & 1.88 \\
\hline 240 & 4.2 & 0.27 & & 0.53 & 0.43 & 1.39 & 0.40 & 0.40 & 1.82 & 1.89 \\
\hline 220 & 4.5 & 0.20 & & 0.57 & 0.43 & 1.41 & 0.39 & 0.39 & 1.67 & 1.87 \\
\hline 200 & 5.0 & 0.18 & & 0.62 & 0.44 & 1.42 & 0.38 & 0.39 & 1.46 & 1.74 \\
\hline 190 & 5.3 & 0.13 & & 0.64 & 0.44 & 1.42 & 0.37 & 0.40 & 1.35 & 1.62 \\
\hline 180 & 5.6 & 0.06 & 0.81 & 0.67 & 0.44 & 1.42 & 0.35 & 0.40 & 1.23 & 1.42 \\
\hline 170 & 5.9 & 0.10 & & 0.70 & 0.44 & 1.42 & 0.34 & 0.39 & 1.17 & 1.27 \\
\hline 165 & 6.1 & 0.09 & & 0.71 & 0.44 & 1.42 & 0.32 & 0.39 & 1.15 & 1.20 \\
\hline 160 & 6.3 & 0.05 & 1.13 & 0.72 & 0.44 & 1.42 & 0.30 & 0.37 & 1.14 & 1.13 \\
\hline 155 & 6.5 & 0.07 & & 0.74 & 0.44 & 1.41 & 0.29 & 0.35 & 1.14 & 1.10 \\
\hline 151 & 6.6 & 0.05 & & 0.75 & 0.44 & 1.42 & 0.27 & 0.32 & 1.14 & 1.11 \\
\hline 145 & 6.9 & 0.05 & 1.13 & 0.76 & 0.44 & 1.42 & 0.25 & 0.28 & 1.12 & 1.13 \\
\hline 140 & 7.1 & 0.05 & 1.29 & 0.77 & 0.44 & 1.42 & 0.24 & 0.25 & 1.05 & 1.10 \\
\hline 135 & 7.4 & 0.05 & 1.38 & 0.78 & 0.44 & 1.43 & 0.23 & 0.24 & 0.95 & 1.02 \\
\hline 130 & 7.7 & 0.05 & 1.34 & 0.79 & 0.44 & 1.43 & 0.21 & 0.23 & 0.83 & 0.90 \\
\hline 125 & 8.0 & 0.03 & 1.25 & 0.79 & 0.44 & 1.44 & 0.17 & 0.19 & 0.69 & 0.72 \\
\hline 120 & 8.3 & 0.04 & 1.14 & 0.80 & 0.45 & 1.44 & 0.13 & 0.12 & 0.55 & 0.57 \\
\hline 115 & 8.7 & 0.06 & 1.41 & 0.81 & 0.45 & 1.45 & 0.08 & 0.05 & 0.43 & 0.43 \\
\hline 110 & 9.1 & 0.09 & 1.79 & 0.82 & 0.45 & 1.46 & 0.06 & 0.04 & 0.34 & 0.34 \\
\hline 100 & 10.0 & 0.16 & 1.42 & 0.82 & 0.46 & 1.48 & 0.04 & 0.03 & 0.24 & 0.23 \\
\hline 90 & 11.1 & 0.27 & 1.68 & 0.82 & 0.46 & 1.50 & 0.04 & 0.03 & 0.25 & 0.24 \\
\hline 80 & 12.5 & 0.19 & 1.41 & 0.81 & 0.48 & 1.54 & 0.07 & 0.04 & 0.39 & 0.39 \\
\hline
\end{tabular}

$a$. From the experimental time constants $\tau_{1}$ in Table 1 .

$b$. From the experimental time constants $\tau_{2}$ in Table $\mathbf{1}$.

c. Calculated by Eq. 1

d. Calculated by Eq. 1. Electronic coupling matrix element $\left(V_{\mathrm{el}}\right)$ was fixed at $50 \mathrm{~cm}^{-1}$.

$e$. Same as $d$ with $V_{\mathrm{el}}=90 \mathrm{~cm}^{-1}$.

$f$. Calculated by Eq. 8-11 with $X(\mathrm{t})=0.5 \times \exp \left(-t / \tau_{\mathrm{s}}\right) ;{ }^{1}$ note that the solvent longitudinal relaxation time $\left(\tau_{\mathrm{L}}\right)$ instead of $\tau_{S}$. in this analysis. ${ }^{2}$ 
Table S2. Temperature Dependent Solvent Densities $(\rho)$, Optical $\left(\varepsilon_{\mathrm{op}}\right)$ and Static $\left(\varepsilon_{\mathrm{s}}\right)$ Solvent Dielectric Constants, Solvent Reorganization Energy $\left(\lambda_{\mathrm{s}}\right)$ for 2-MTHF, Along with the Computed Reaction Free Energy $(\Delta G)$ for the 2a-Zn Photoinduced CS Reaction.

\begin{tabular}{|c|c|c|c|c|c|c|}
\hline $\begin{array}{c}\mathrm{T} \\
(\mathrm{K})\end{array}$ & $\begin{array}{c}1000 / \mathrm{T} \\
\left(\mathrm{K}^{-1}\right)\end{array}$ & $\begin{array}{c}\rho^{a} \\
\left(\mathrm{~g} \mathrm{~cm}^{-1}\right)\end{array}$ & $\varepsilon_{\mathrm{op}}{ }^{b}$ & $\varepsilon_{\mathrm{s}}^{c}$ & $\begin{array}{c}\lambda_{\mathrm{s}}{ }^{d} \\
(\mathrm{eV})\end{array}$ & $\begin{array}{c}\Delta G^{e} \\
(\mathrm{eV})\end{array}$ \\
\hline 320 & 3.1 & 0.823 & 1.94 & 6.58 & 0.481 & -0.808 \\
\hline 310 & 3.2 & 0.833 & 1.95 & 6.73 & 0.480 & -0.814 \\
\hline 290 & 3.4 & 0.852 & 1.98 & 7.06 & 0.479 & -0.829 \\
\hline 280 & 3.6 & 0.862 & 2.00 & 7.25 & 0.479 & -0.836 \\
\hline 270 & 3.7 & 0.872 & 2.01 & 7.47 & 0.480 & -0.844 \\
\hline 260 & 3.8 & 0.882 & 2.03 & 7.70 & 0.480 & -0.853 \\
\hline 240 & 4.2 & 0.902 & 2.06 & 8.25 & 0.481 & -0.870 \\
\hline 220 & 4.5 & 0.922 & 2.09 & 8.95 & 0.484 & -0.890 \\
\hline 200 & 5.0 & $0.942^{f}$ & 2.12 & 9.87 & 0.488 & -0.911 \\
\hline 190 & 5.3 & $0.953^{\mathrm{f}}$ & 2.14 & 10.45 & 0.490 & -0.923 \\
\hline 180 & 5.6 & $0.963^{f}$ & 2.16 & 11.14 & 0.493 & -0.935 \\
\hline 170 & 5.9 & $0.973^{f}$ & 2.18 & 11.97 & 0.497 & -0.947 \\
\hline 165 & 6.1 & $0.978^{f}$ & 2.18 & 12.45 & 0.498 & -0.954 \\
\hline 160 & 6.3 & $0.984^{\mathrm{f}}$ & 2.19 & 12.99 & 0.500 & -0.961 \\
\hline 155 & 6.5 & $0.989^{f}$ & 2.20 & 13.59 & 0.503 & -0.968 \\
\hline 151 & 6.6 & $0.993^{f}$ & 2.21 & 14.13 & 0.504 & -0.974 \\
\hline 145 & 6.9 & $0.999^{f}$ & 2.22 & 15.04 & 0.507 & -0.982 \\
\hline 140 & 7.1 & $1.005^{\mathrm{f}}$ & 2.23 & 15.94 & 0.510 & -0.990 \\
\hline 135 & 7.4 & $1.010^{\mathrm{f}}$ & 2.24 & $16.97^{\mathrm{g}}$ & 0.512 & -0.998 \\
\hline 130 & 7.7 & $1.015^{\mathrm{f}}$ & 2.25 & $18.19^{\mathrm{g}}$ & 0.515 & -1.006 \\
\hline 125 & 8.0 & $1.020^{\mathrm{f}}$ & 2.26 & $19.64^{\mathrm{g}}$ & 0.518 & -1.014 \\
\hline 120 & 8.3 & $1.026^{\mathrm{f}}$ & 2.27 & $21.40^{\mathrm{g}}$ & 0.521 & -1.023 \\
\hline 115 & 8.7 & $1.031^{\mathrm{f}}$ & 2.28 & $23.58^{\mathrm{g}}$ & 0.524 & -1.032 \\
\hline 110 & 9.1 & $1.036^{\mathrm{f}}$ & 2.28 & $26.34^{\mathrm{g}}$ & 0.528 & -1.041 \\
\hline 100 & 10.0 & $1.047^{\mathrm{f}}$ & 2.30 & $34.95^{\mathrm{g}}$ & 0.535 & -1.060 \\
\hline 90 & 11.1 & $1.058^{\mathrm{f}}$ & 2.32 & $53.69^{\mathrm{g}}$ & 0.544 & -1.078 \\
\hline 80 & 12.5 & $1.069^{\mathrm{f}}$ & 2.34 & $125.96^{\mathrm{g}}$ & 0.553 & -1.102 \\
\hline
\end{tabular}

$a$. Determined using the relation $\rho(T)=1157-1.124 \cdot T+2.54 \times 10^{-4} \bullet T^{2}$.

$b$. Estimated using the Lorenz-Lorentz expression ref for the molar refractivity, $\rho$, which is

$$
\rho(T)=\left(\frac{\varepsilon_{o p}(T)-1}{\varepsilon_{o p}(T)+2}\right) \frac{M}{d_{20}}
$$

where $M$ is the molecular weight and $d$ is the density at the $20{ }^{\circ} \mathrm{C} .{ }^{4}$

$c$. Estimated by the equation obtained by Furutsuka et. al. ${ }^{5}$

$\varepsilon_{s}(T)=3.1+\frac{860}{T-73}$

$d$. Solvent reorganization energy calculated by dielectric continuum model modified by Tachiya. ${ }^{6}$ Overall molecular shape is assumed to be a cylinder with $4.9 \AA$ diameter and $10.61 \AA$ length $\left(2 \mathrm{c}+R_{\mathrm{DA}}\right.$, where $\mathrm{c}$ is the half of the van der Waals thickness $\left(1.75 \AA\right.$ ) of porphyrin or quinone and $R_{\mathrm{DA}}$ is the porphyrin plane-to-quinonyl centroid distances (7.11 $\AA$ ) for DFT optimized structures). ${ }^{7}$ 
e. Driving force calculated by Weller's equation (Eq. 4)

$f$. The equation in footnote $a$ is derived from the data above $200 \mathrm{~K}$. The listed values are obtained by extrapolation. $g$. In ref 5, the magnitudes of are 19.5 at $115 \mathrm{~K}$ and $2.6 \pm 0.1$ at $<90 \mathrm{~K}$, indicating an abrupt change of dielectric behavior $\sim 100 \mathrm{~K}$. ET rate constants with and calculated by these values showed huge decrease below $90 \mathrm{~K}$, which was not observed in the experiment.

\section{References and Notes}

1. Ushakov, E. N.; Nadtochenko, V. A.; Gromov, S. P.; Vedernikov, A. I.; Lobova, N. A.; Alfimov, M. V.; Gostev, F. E.; Petrukhin, A. N.; Sarkisov, O. M. Chem. Phys. 2004, 298, 251-261.

2. As a CT absorption band is not observed for $2 \mathrm{a}-\mathrm{Zn}$, the nuclear reorganization energy for semiclassical model was partitioned into $\lambda_{\mathrm{cl}, \mathrm{vib}}=0.15 \mathrm{eV}, \lambda_{\mathrm{v}}=0.35 \mathrm{eV}$.

3. Shinsaka, K.; Gee, N.; Freeman, G. R. J. Chem. Thermodyn. 1985, 17(12), 1111-1119.

4. Khundkar, L. R.; Perry, J. W.; Hanson, J. E.; Dervan, P. B. J. Am. Chem. Soc. 1994, 116, 9700-9709.

5. Furutsuka, T.; Imura, T.; Kojima, T.; Kawabe, K. Technology Reports of the Osaka University 1974, 24, 367-369.

6. Kato, T.; Tachiya, M. Chem. Phys. Lett. 1995, 241, 463-468.

7. Zheng, J. R.; Kang, Y. K.; Therien, M. J.; Beratan, D. N. J. Am. Chem. Soc. 2005, 127, 11303-11310. 